\title{
ON STATISTICALLY KÖTHE-TOEPLITZ DUALS
}

\author{
FAtih Temizsu And Mikail Et
}

Abstract. In the present paper, we introduce the concept of $\Delta^{m}$-statistical boundedness of real (or complex) numbers sequences by using generalized difference operator $\Delta^{m}$ and examine relationships between $\Delta^{m}$ - statistical convergence, $\Delta^{m}$-statistical Cauchiness and $\Delta^{m}$-statistical boundedness. In addition to that we compute the Köthe-Toeplitz and generalized Köthe-Toeplitz duals of the set of all $\Delta^{m}$ - statistical bounded sequences. Moreover, we come up with the idea of statistical $\alpha$ and $\beta$ duals of the sets of sequence which makes us capable of creating statistical equivalents of the notions of normality and perfectness of sequence spaces. Mathematics subject classification (2010): $40 \mathrm{~A} 05,40 \mathrm{C} 05,40 \mathrm{~A} 45$.

Keywords and phrases: Statistical boundedness, dual spaces.

\section{REFERENCES}

[1] Bektaş, Ç. A., Et, M. And ÇOLAK, R., Generalized difference sequence spaces and their dual spaces, J. Math. Anal. Appl. 292(2) (2004), 423-432.

[2] Bhardwaj, V. K. And Gupta, S., On some generalizations of statistical boundedness, J. Inequal. Appl., 2014:12 (2014).

[3] Boos, J., Classical and Modern Methods in Summability, Oxford University Press, Oxford (2000).

[4] Connor, J. S., The statistical and strong p-Cesàro convergence of sequences, Analysis 8 (1988), $47-63$.

[5] Connor, J. S., On strong matrix summability with respect to a modulus and statistical convergence, Canad. Math. Bull. 32(2) (1989), 194-198.

[6] ET, M. AND ÇOLAK, R., On some generalized difference sequence spaces, Soochow J. Math. 21(4) (1995), 377-386.

[7] Et, M. and Nuray, F., $\Delta^{m}$-Statistical Convergence, Indian J. Pure Appl. Math., 32(6) (2001), 961-969.

[8] Et, M., Altinok, H. And Altin, Y., On some generalized sequence spaces, Appl. Math. Comput. 154(1) (2004), 167-173.

[9] Et, M., MurS AleEn, M. AND IşıK, M., On a class of fuzzy sets defined by Orlicz functions, Filomat 27(5) (2013), 789-796.

[10] FAST, H., Sur la convergence statistique, Colloquium Math. 2 (1951), 241-244.

[11] FRIDY, J. A., On statistical convergence, Analysis 5 (1985), 301-313.

[12] Fridy, J. A. AND ORHAN, C., Statistical limit superior and limit inferior, Proc. Amer. Math. Soc. 125(12) (1997), 3625-3631.

[13] Kampthan, P. And Gupta, M., Sequence spaces and series, Lecture Notes in Pure and Applied Mathematics, 65. Marcel Dekker, Inc., New York (1981).

[14] Kizmaz, H., On certain sequence spaces, Canad. Math. Bull. 24(2) (1981), 169-176.

[15] Steinhaus, H., Sur la convergence ordinaire et la convergence asymptotique, Colloquium Mathematicum 2 (1951), 73-74.

[16] Temizsu, F., Et, M. And ÇInAR, M., $\Delta^{m}$-deferred statistical convergence of order $\alpha$, Filomat 30(3) (2016), 667-673.

[17] Tripathy, B. C., On statistical convergence, Proc. Estonian Acad. Sci. Phys. Math. 47 (1998), 299303.

[18] Tripathy, B. C., On statistically convergent series, Punjab University J. Math. XXXXII, (1999), $1-8$. 